\title{
Multispectral linear filtering of high resolution EELS images by geostatistics
}

\author{
Pinnamaneni Bhanu Prasad $\left(\left(^{1,2, *}\right)\right.$, Dominique Jeulin $\left({ }^{1}\right)$, Colin Daly $\left({ }^{1}\right)$, Claudie Mory $\left({ }^{3}\right)$, \\ Marcel Tencé $\left({ }^{3}\right)$ and Christian Colliex $\left({ }^{3}\right)$
}

( $\left.{ }^{1}\right)$ Centre de Géostatistique, Ecole des Mines, 35 Rue Saint Honoré, 77305 Fontainebleau, France

${ }^{2}$ ) LERMAT, ISMRa, Université de Caen, 14032 Caen Cédex, France

$\left({ }^{3}\right)$ Laboratoire de Physique des Solides associé au CNRS, Bâtiment 510, Université Paris-Sud, 91405 Orsay Cedex, France

(Received December 12, 1990; accepted March 05, 1991)

\begin{abstract}
Elemental mapping obtained from EELS spectra suffers from a relatively poor signal/noise ratio due to low counting statistics when looking for the ultimate spatial resolution of the instrument. This paper is devoted to the application of geostatistical techniques for filtering EELS images: multivariate optimal linear filters improve the $S / N$ ratio by a factor 5 to 10 in the case of uranium and terbium bright field images, without apparent loss of spatial resolution. A systematic use of this method applied to EELS images would help progressing in the direction of single atom identification with this technique.
\end{abstract}

\section{Introduction.}

Elemental mapping with high spatial resolution can be achieved by processing sequences of energy filtered images, recorded at energy losses below and above the ionization edges of the involved elements. Two instrumental configurations are used to provide energy filtered images: a) the conventional electron microscope equipped with a filter (generally of the Castaing-Henry type [1] introduced in the column); b) the scanning transmission electron microscope, in which case the filtered image is sequentially recorded by scanning a focused beam over the specimen and by detecting the energy loss electrons of interest through a selection slit at the exit plane of a magnetic analyser (the EELS spectrometer) - see [2,3] for a general description. This mode has been used for the present study.

In an EELS spectrum, the characteristic signal due to a core level excitation is superimposed over a non-characteristic background. Hence a single energy filtered image does not constitute a useful map of the spatial distribution of the chemical element. As proposed by Jeanguillaume et al. [4], it is essential to record a sequence of energy filtered images and to extrapolate, for each

$\left(^{*}\right)$ Presently: Confocal Technologies Ltd, Mulberry Place, Liverpool L7 7HJ, U.K. 
pixel, the information contained in the energy channels before the edge. One can then discriminate the characteristic signal from the background in the intensity measured for an energy loss above the edge. The useful methodology has been developed by different authors: for instance, Bonnet et al. [5] have discussed several pertinent aspects of background modelization and proposed simple routes with standard smoothing procedures in order to improve the signal to noise ratio in elemental maps.

As a matter of fact, one major limitation when pushing the technique to its limits, i.e. to the potential identification of atomic clusters (see Mory et Colliex [6], Mory et al. [7]), lies in the relatively weak signal to noise ratio. For reasonable total recording times, the dwell-time per pixel and energy loss must remain in the millisecond range. When one is concerned with core-loss events of weak cross sections (typically of the order of $10^{-21}-10^{-20} \mathrm{~cm}^{2} /$ atom for an energy window of about $10 \mathrm{eV}$ above the edge), high flux of primary electrons are required. They are generally delivered from a field emission gun into a subnanometer probe. Nevertheless, the total number of counts in a characteristic image is very low (generally a few hundred). Typical examples of such noisy images resulting in poor elemental maps in terms of signal to noise ratio are shown in this paper together with numerical data. It is therefore of prime importance to develop efficient restoration methods on such sequences of images in order to improve the signal to noise ratio for the extraction of a quantitative elemental information. Local treatments of the raw data are necessary and it is advisable to chose optimal filtering techniques so that the original signal and the point resolution are not disturbed. The purpose of the present paper is to apply on practical situations where EELS elemental mapping is pushed at its limits, methods originally developed in geostatistics.

These involve an optimal linear filter often called a Wiener filter [8] in the literature on signal or image processing [9]. The originality of our approach resides in the following points: generalization to multivariate images, applicability to non stationary data, and implementation of coherent multivariate variogram models, which are not chosen a priori but are estimated from the images.

In parallel to this work, Trebbia et al. [10] have followed another approach, the factorial analysis of correspondence, for processing the same series of original data.

\section{EELS instrumentation for data acquisition.}

The experiments have been made with a dedicated STEM (Vacuum Generators HB 501) delivering on the specimen entrance surface $100 \mathrm{kV}$ incident electrons within a probe of nominal diameter $\simeq 0.5 \mathrm{~nm}$. As shown in figure 1 several detectors measure the different contributions on the transmitted beam. An annular dark field detector collects all electrons scattered mostly through elastic processes to large angle. The forward beam is analysed with a Gatan magnetic spectrometer and the electron energy loss spectrum is scanned in front of a selection slit and recorded with a standard scintillator - photomultiplier detector (BF detector). Each BF image depends upon the energy loss selected and hence is identified as an energy filtered image by the energy loss utilised $\left(E_{1}, E_{2} \ldots\right)$. For each position of the probe on the specimen, one simultaneously records an $\mathrm{ADF}$ signal $\left(I_{\mathrm{el}}\right)$ and an inelastic signal with energy loss $E$, i.e. $I(E)$. Image acquisition is under digital control. A specific interface has been elaborated and built in order to address successively different energy losses and to record $I_{1}, \ldots I_{N}$. It is also used to scan the probe on the specimen. As a consequence, one obtains within one global image scan a sequence of $N$ inelastic images with the BF detector, and $N$ annular dark field (ADF) images which are simultaneously acquired. The inelastic images are a-posteriori processed following the algorithms described in [5]. It models the background before the edge with a mathematical law such as $A . E^{-R}$. The values of $A$ and $R$ are estimated by regression from the content of the images $I_{1}, I_{2} \ldots$ before the edge using the 
equation: $\log I=\log A-R \log E$. The extrapolation to the energy loss $\bar{E}$ under the edge gives $B$ and the characteristic signal is finally estimated as: $S=I-B$.

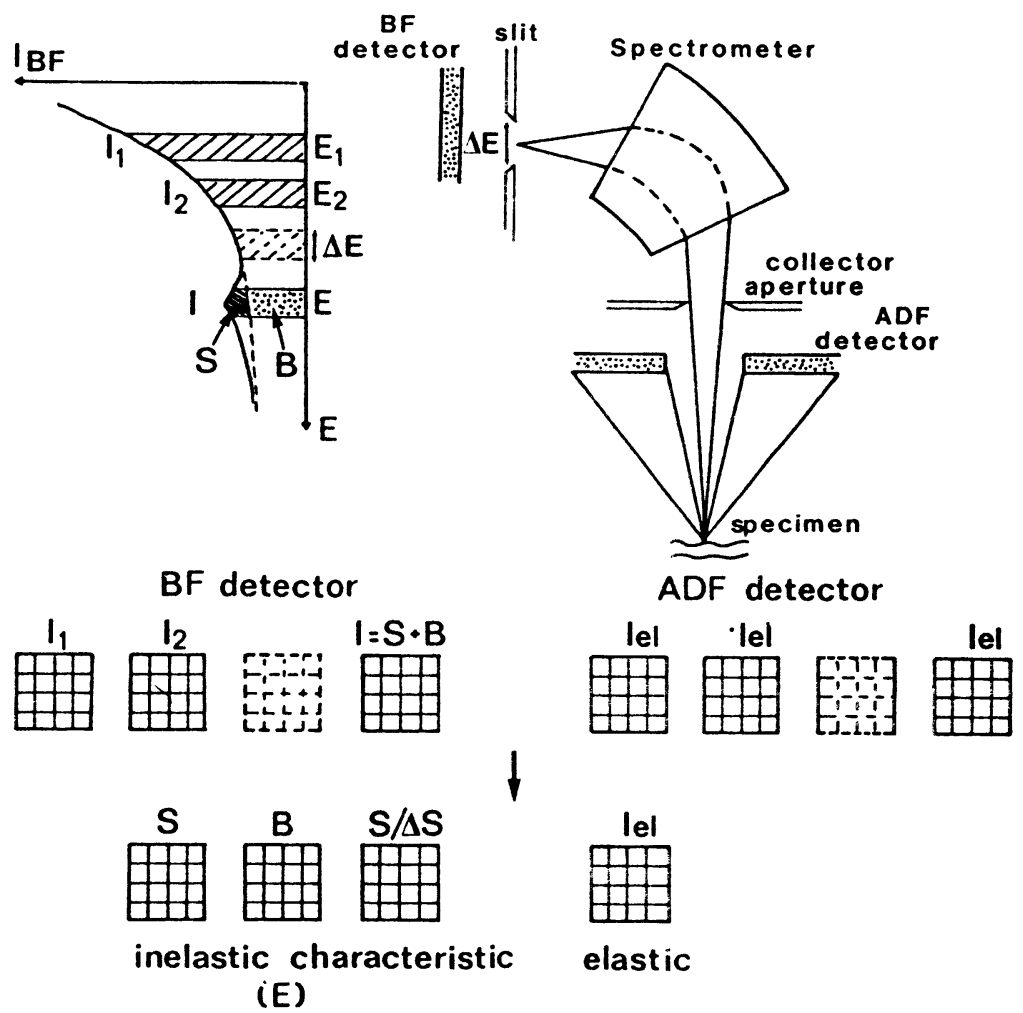

Fig. 1. - Multiple signal acquisition with a STEM. The ADF detector collects all electrons scattered to large angles $\left(I_{\mathrm{el}}\right)$. The BF detector at the spectrometer exit collects all electrons transmitted with a given energy loss $E_{i}$ defined by the excitation of the magnet and the selection slit. The sequence of energy loss images $I_{1}, I_{2} \ldots I$ has to be processed in order to discriminate in the image recorded above the ionization edge, the characteristic information $\mathrm{S}$ from the background contribution $\mathrm{B}$.

Another useful feature is the possibility of duplicating any type of image, in which case the signal is the same and the noise content is different. The observed data may then be considered as a randomisation of the underlying variable and the duplicated pair of images can be utilised to estimate, by cross-correlation techniques, the underlying structure of the variable.

\section{Results: images of clusters.}

Two specimens consisting of randomly scattered clusters of heavy metal on thin supporting layers have been prepared by E. Delain (Inst. G. Roussy, Villejuif) following the standard techniques of positively staining isolated biomolecules (DNA, RNA, filaments...) - see for instance [11]. The used salts are uranyl acetate, which gives uranium clusters with sizes ranging from a single atom up to particles of 2-3 nm (made of a few hundred atoms) and terbium chloride for terbium clusters. 
3.1 Uranium images. - As shown in spectrum 2a, the useful edge is the $\mathrm{O}_{45}$ one at about $100 \mathrm{eV}$. Consequently energy filtered images were acquired at different energy losses below the edge (59, $74,89 \mathrm{eV}$ ) and the final one at $112 \mathrm{eV}$, which is about the maximum of the characteristic uranium signal. As a matter of fact, each BF image has been duplicated at energy losses slightly higher $(59.2,74.2,89.2$ and $112.2 \mathrm{eV})$. Practically, the raster scan consists of $128 \times 128$ pixels with a pixel step of $0.2 \mathrm{~nm}$ at a direct magnification of 4 million. The probe diameter is about $0.5 \mathrm{~nm}$, so that the image is slightly oversampled. The intensities are digitalised using a 16 bits range. The images (see Fig. 3, showing the average of two acquisitions, before and after filtering) contain a stretch of DNA filament with high intensity values. The characteristic uranium map was calculated with the described routine. The numerical characteristics of the different images are summarized in table I.
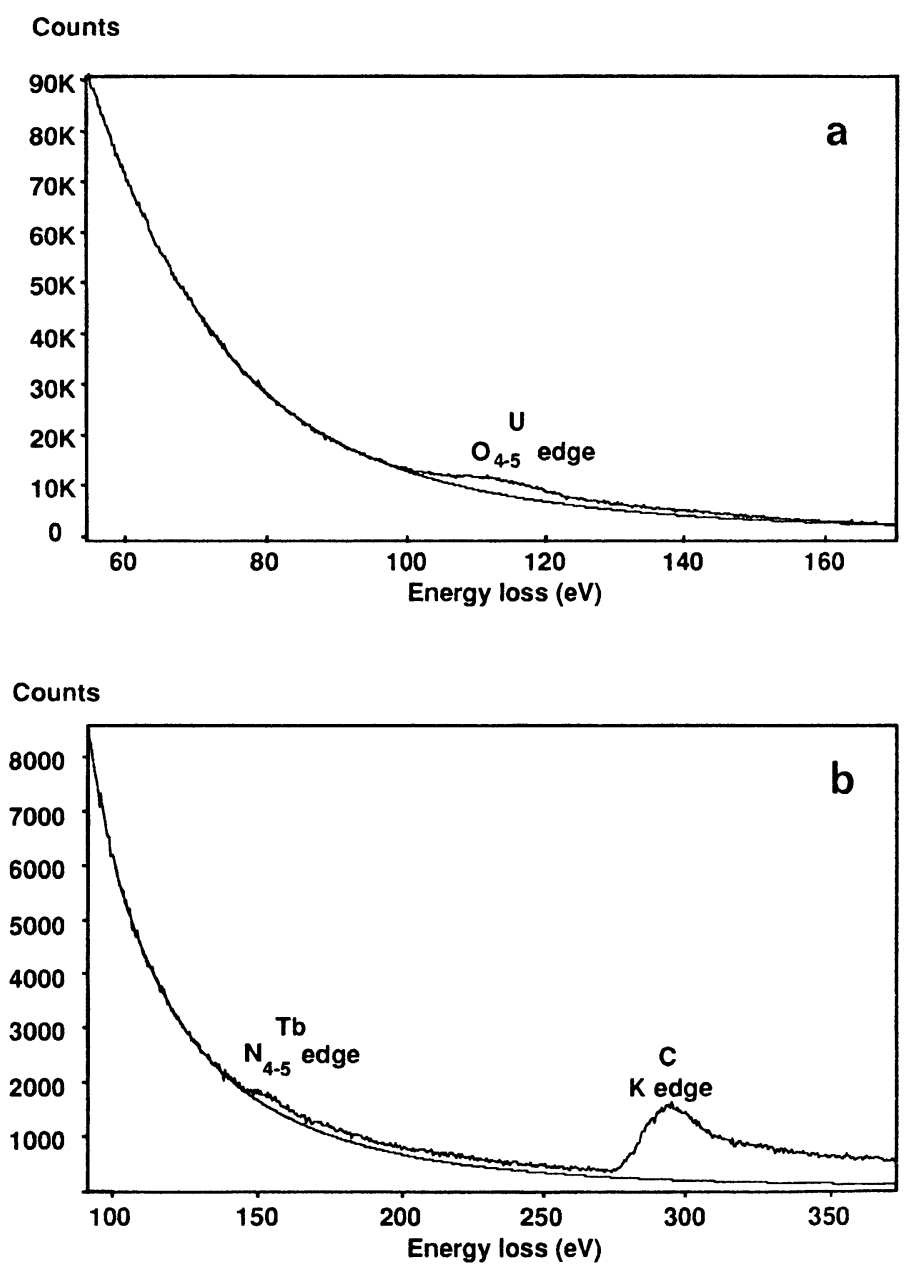

Fig. 2. - Typical spectra showing the edges $\mathrm{U}-\mathrm{O}_{45}$ (a) and $\mathrm{Tb}-\mathrm{N}_{45}$ (b) used for elemental mapping of small uranium and terbium clusters deposited on a thin amorphous carbon foil. They have been recorded with opened selection slits, as used for recording the sequences of energy filtered images. 

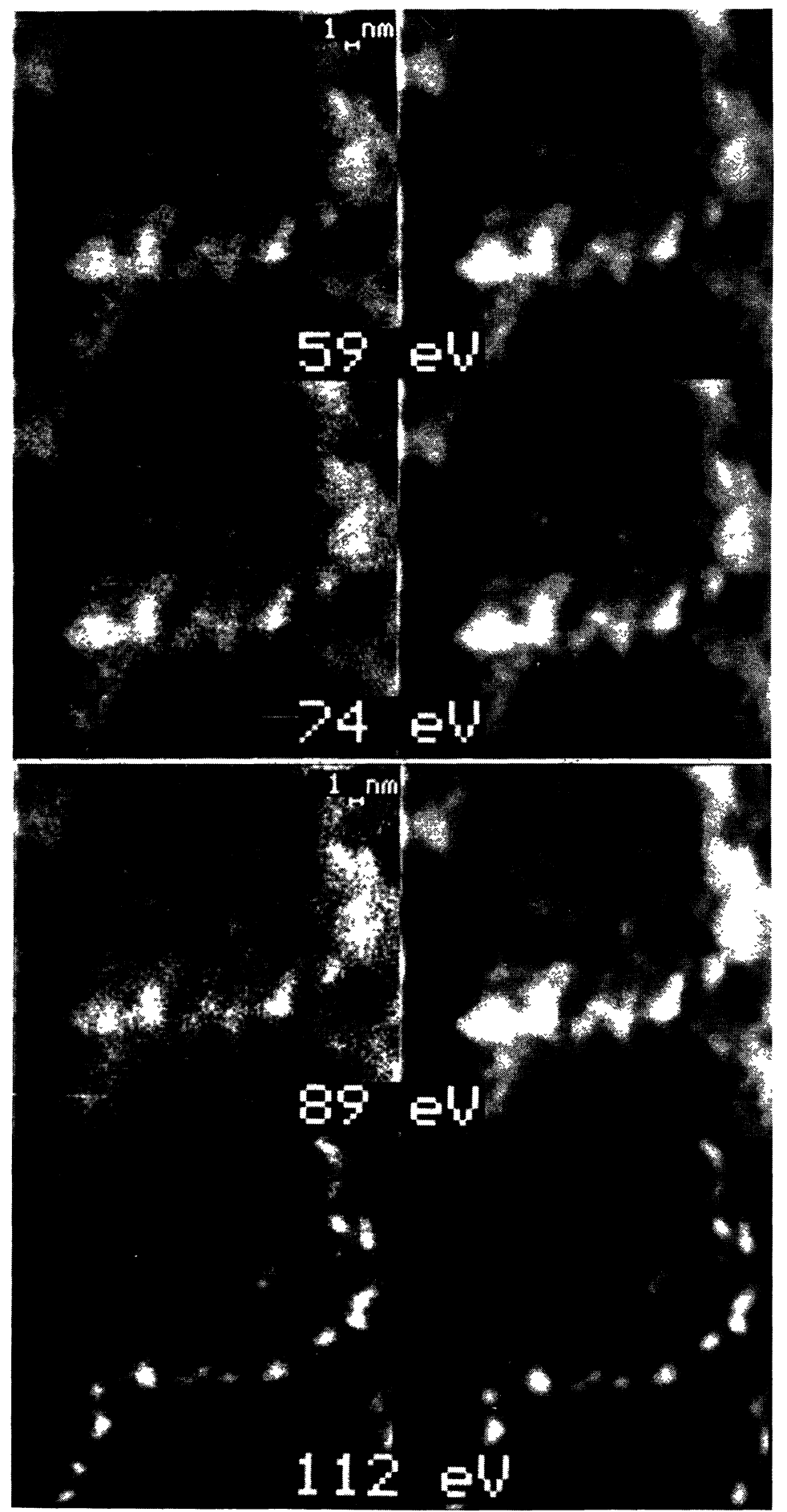

Fig. 3. - Sequences of energy filtered $\mathrm{BF}$ images for the extraction of the characteristic uranium $\mathrm{O}_{45}$ signal at $\simeq 105 \mathrm{eV}$. Original images are in the left column and the corresponding filtered images (see further) are in the right column. 
Table I. - Statistics of the series of U images (cf. Fig. 3, left column).

\begin{tabular}{|l|c|c|c|c|c|}
\hline Image $\rightarrow$ & BF 59 & BF 74 & BF 89 & BF 112 & BF CHA \\
\hline Mean & 4169 & 2059 & 1123 & 666 & 113 \\
Variance & 133542 & 36245 & 12353 & 11393 & 9319 \\
Minimum & 3267 & 1579 & 809 & 420 & 67 \\
Maximum & 5430 & 2728 & 1488 & 1478 & 208 \\
\hline
\end{tabular}

3.2 Terbium images. - The useful edge is the terbium $\mathrm{N}_{45}$ edge at about $145 \mathrm{eV}$ (see Fig. $2 \mathrm{~b}$ ). Consequently, energy filtered images have been acquired at 131, 141 and $151 \mathrm{eV}$. The first two are used for background modeling and the last one contains the characteristic $\mathrm{Tb}$ signal which can be computed similarly to the previous case. Figure 4 shows original images (average of two acquisitions, before and after filtering). Their statistical content is gathered in table II.

Table II. - Statistics of the series of Tb images.

\begin{tabular}{|l|c|c|c|}
\hline \multicolumn{1}{|c|}{ Image } & BF 131 & BF 141 & BF 151 \\
\hline & Original & Original & Original \\
Mean & 117.14 & 92.42 & 76.02 \\
Variance & 143.76 & 98.30 & 74.52 \\
Minimum & 81 & 61 & 48 \\
Maximum & 161 & 131 & 110 \\
\hline
\end{tabular}

The histograms have been calculated and the pixels with extreme values - they have been found more numerous at the end of the histogram than in the beginning - have been masked. This procedure, already proposed in [14], is required to avoid to disturb the variograms by the occurrence of large extreme values. Consequently, the range of intensity for the BF 131 image is reduced from (67-229) to (81-161). The other images have been masked in a similar way and the global mask has been selected as the union of all these masks.

\section{Linear optimal filtering from geostatistical models.}

The occurrence of noise in images requires the use of statistical models and methods to improve the observed signal. In this section we briefly introduce the geostatistical technique used to estimate the underlying data from the observed images. In particular we emphasize the optimal linear filtering of the data, namely the kriging procedure developed for estimating unknown or noisy data $[12,13]$. In our case, multivariate filters are developed following the methodology proposed for microprobe images [14].

Consider that the data consist of $N$ images (variables) $Z_{i}(x)$ obtained at pixeis $x$. In the present case, we operate on the available bright field images $Z_{i}(i=1,2 \ldots N)$ obtained on the same specimen area (multivariate case). We can either work on a single image $Z(x)$. namely a single bright field or a characteristic image. The observed images $Z_{i}(x)$ are spoiled by noise, so that the underlying (pure) signal $Y_{i}(x)$ is not available. Our filtering procedure applied to the average of two 


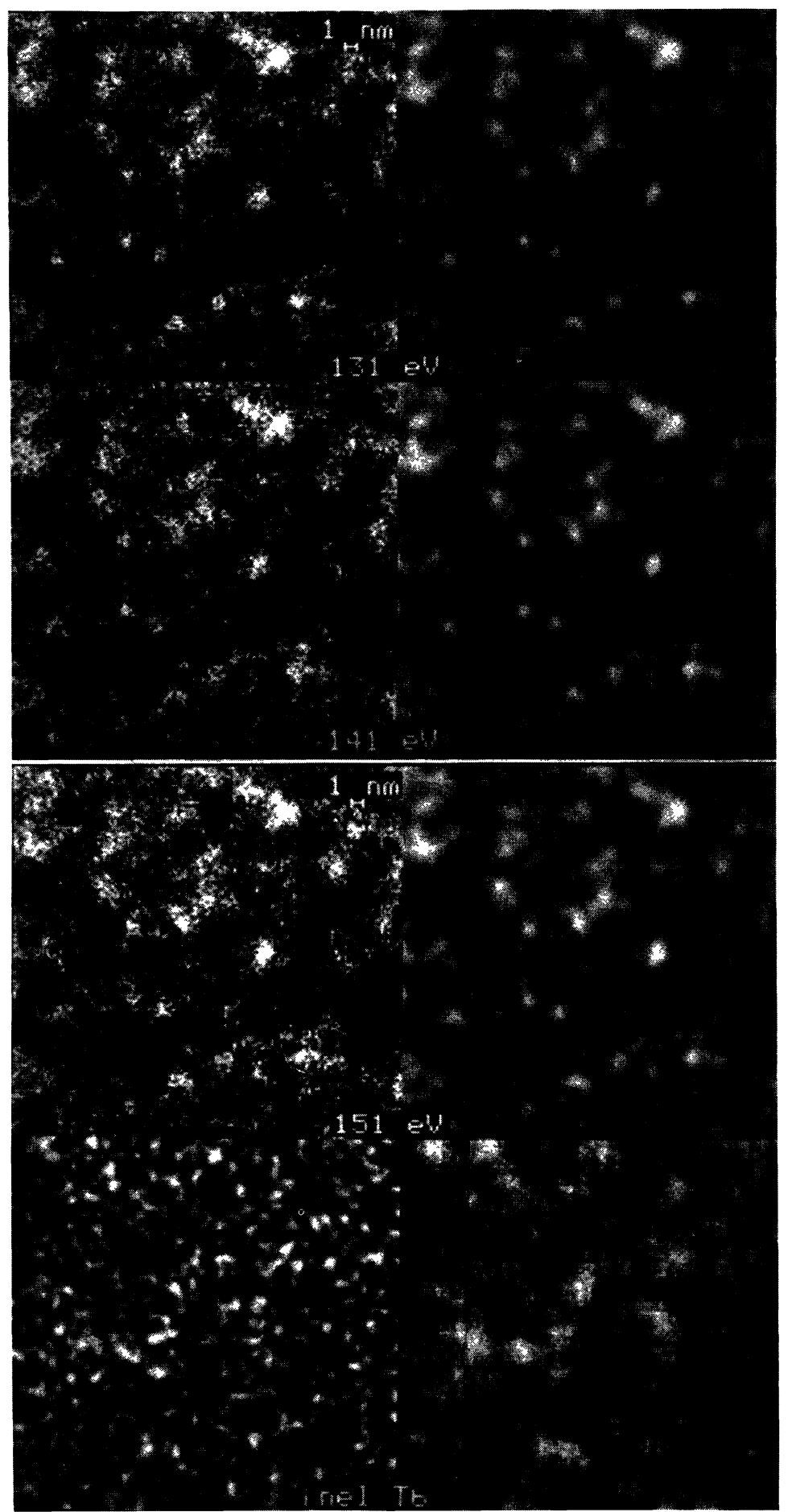

Fig. 4. - Sequences of energy filtered images for the extraction of the characteristic terbium $\mathrm{N}_{45}$ signal at $\simeq 148 \mathrm{eV}$. Original images are in the left column and the corresponding filtered images (see further paragraph 5.2) are in the right column. 
acquisitions aims to produce an estimator $y_{i}(x)$ of each $Y_{i}(x)$ given by the observed data $Z_{i}(x)$ at different pixels. As these filters are linear, we calculate a linear combination of the multivariate data $Z_{i}(x)$ in a given neighbourhood of each pixel. The coefficients of the filters are the coefficients of the linear regression of the unknown $y_{i}(x)$, from the available data $\left.Z_{i}(x)\right)$. The filters are optimal, since they minimize the variance of the estimation. To solve this problem, we need first to introduce the notion of variogram. The reader who is not interested in the details of this approach can skip the rest of section 4.

4.1 VARIOGRAMS. - Let us consider both the observed variables $Z_{i}(x)$ and the corresponding underlying variable $Y_{i}(x)$ to have second order stationary increments $\left(Z_{i}\left(x_{1}\right)-Z_{i}\left(x_{2}\right)\right)$ : the mean and the covariance of the increments are finite and independent of location. This can be expressed as ( $E$ representing the mathematical expectation, in practice, the average value over the couples $x_{1}$ and $x_{2}$ ):

$$
E\left[Z_{i}\left(x_{1}\right)-Z_{i}\left(x_{2}\right)\right]
$$

and

$$
\gamma_{i j}(h)=\frac{1}{2} E\left[\left(Z_{i}\left(x_{1}\right)-Z_{i}\left(x_{2}\right)\right)\left(Z_{j}\left(x_{1}\right)-Z_{j}\left(x_{2}\right)\right)\right]
$$

are independent of $x_{1}$ and $x_{2}$ for all $i, j=1 \ldots N$ and depend only on the distance $h=\left|x_{1}-x_{2}\right|$. For $i=j$, we have:

$$
\gamma_{i i}(h)=\frac{1}{2} E\left[\left(Z_{i}\left(x_{1}\right)-Z_{i}\left(x_{2}\right)\right)^{2}\right]
$$

The $\gamma_{i j}(i \neq j)$ are called cross variograms and $\gamma_{i i}$ are simple variograms. In practice, the experimental variogram is calculated from:

$$
\gamma_{i i}(h)=\frac{1}{2 N_{\mathrm{p}}} \sum_{N_{\mathrm{p}}}\left[Z_{i}(x)-Z_{i}(x+h)\right]^{2}
$$

and

$$
\gamma_{i j}(h)=\frac{1}{2 N_{\mathrm{p}}} \sum_{N_{\mathrm{p}}}\left[\left(Z_{i}(x)-Z_{i}(x+h)\right)\left(Z_{j}(x)-Z_{j}(x+h)\right)\right]
$$

where $N_{\mathrm{p}}$ is the number of pairs $(x, x+h)$.

Where the underlying data $Y_{i}(x)$ are modelled by second order stationary random functions, the variogram is related to the covariance function $C_{i j}(h)$ by:

$$
\begin{gathered}
C_{i j}(h)=E\left[Z_{i}(x) Z_{j}(x+h)\right] \\
\gamma_{i j}(h)=C_{i j}(0)-C_{i j}(h)
\end{gathered}
$$

We shall see later how the variogram function is involved in the design of optimal filters. In addition it conveys the structural information on the coregionalisation under study. The observed variograms obtained for uranium and terbium EELS images are presented in figures 5 and 6 . In both cases a superposition of various scales in the structure is apparent from points of inflexion on the experimental variograms. For instance a 10 to 12 pixel range corresponding to the average diameter of uranium atom clusters is seen in figure 5. It should be noticed that the variograms are discontinuous near the $h=0$. In the present case this fact relates to the presence of noise in the image. The discontinuity (called nugget effect) is the variance of the noise while the sill of the variogram is the total variance (signal+noise). In addition, when a component of the microstructure has a range lower than the smallest distance between 2 pixels, its own variance contributes to the nugget effect. In most cases, the sill is reached by the variogram for a finite distance $a$, called the range. It measures a characteristic length of the microstructure: for two pixels $x_{1}$ and $x_{2}$ separated by a distance larger than $a$, the data $Z_{i}\left(x_{1}\right)$ and $Z_{j}\left(x_{2}\right)$ are uncorrelated. 



Fig. 5a. - Simple (left) and cross (right) observed variograms for the uranium BF images in group 1 (i.e. for images recorded below the $U$ edge). 




b)

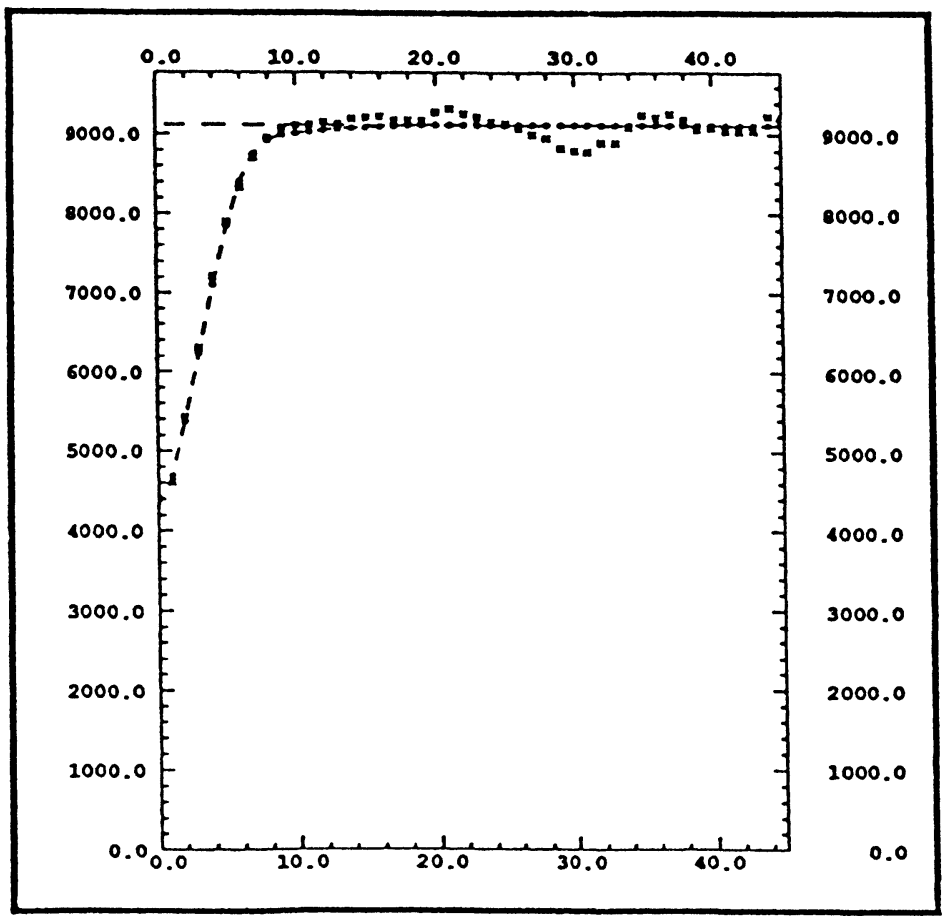

c)

Fig. 5b,c. - b) Observed variogram for the BF image at $112 \mathrm{eV}$ (i.e. in group 2, above the U edge). c) Observed variogram for the processed characteristic uranium image (i.e. in group 3 ). The horizontal scale is calibrated in distances between pixels. 

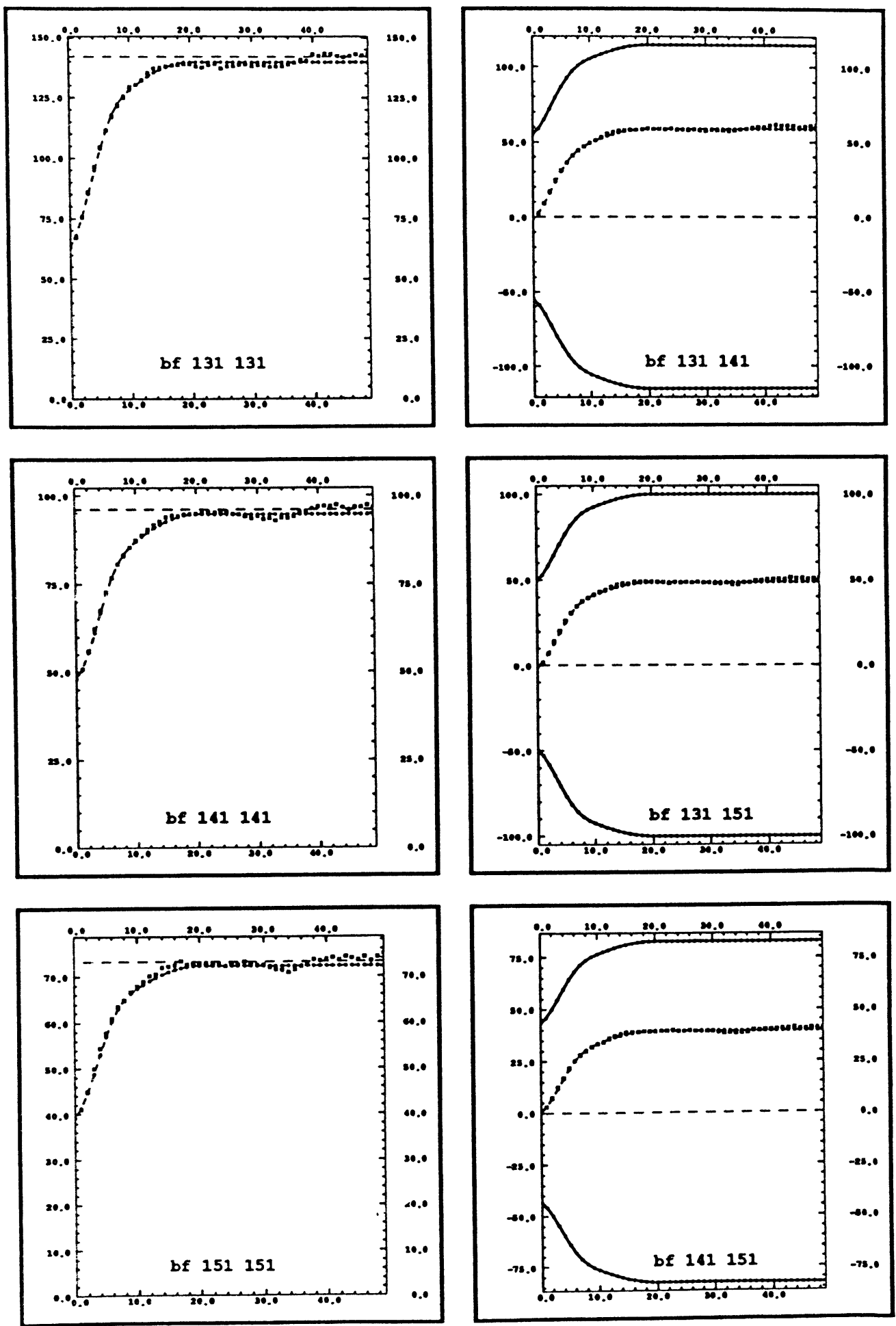

Fig. 6. - Simple (left) and cross (right) variograms for the energy filtered images in the terbium case. 
4.2 KRIGING SYSTEM. - To estimate the underlying image $Y_{j}\left(x_{0}\right)$ of the $j^{\text {th }}$ variable at pixel $x_{0}$ using the available observed information (variables $Z_{i}\left(x_{\alpha}\right), \alpha=1,2, \ldots n ; i=1 \ldots N: N$ variables in a neighbourhood of $x_{0}$ with size $n$ ) we use a linear estimator $Y_{j}^{*}\left(x_{0}\right)$ :

$$
Y_{j}^{*}\left(x_{0}\right)=\sum_{\alpha=1}^{n} \sum_{i=1}^{N} \lambda_{i j}^{\alpha} Z_{i}\left(x_{\alpha}\right)
$$

The weights $\lambda_{i j}^{\alpha}$ are the elements of the unbiased optimal filter when the estimator is constructed based on the following 2 conditions:

- unbiasedness: $E\left[Y_{j}(x)-Y_{j}^{*}(x)\right]=0$ which yields the following $N$ conditions on the weigths:

$$
\sum_{\alpha} \lambda_{i j}^{\alpha}=\delta_{i j} \quad \forall i, j=1, \ldots, N
$$

with $\delta_{i j}=1$ if $i=j$, else 0 .

- optimatility condition: the estimation variance is minimum, i.e. $\operatorname{var}\left[Y_{j}(x)-Y_{j}^{*}(x)\right]$ is minimum.

Then the $N^{2}(n+1)$ kriging equations to filter the $j^{\text {th }}$ variable can be written as:

$$
\begin{gathered}
\sum_{i=1}^{N} \sum_{\alpha=1}^{n} \lambda_{i j}^{\alpha} \gamma_{i k}^{\mathrm{o}}\left(x_{\alpha}-x_{\beta}\right)+\mu_{k j}=\gamma_{k j}^{\mathrm{u}}\left(x_{\beta}-x_{\mathrm{o}}\right) \\
\sum_{\alpha=1}^{n} \lambda_{i j}^{\alpha}=\delta_{i j}
\end{gathered}
$$

for all $k=1 \ldots N$ and $\beta=1, \ldots n$. The weights $\lambda_{i j}^{\alpha}$ resulting from the kriging equations are equivalent to the well known Wiener filter used in signal processing. The $\gamma_{i k}^{\mathrm{u}}$ is the underlying variogram, i.e. the variogram of $Y_{i}$ and $Y_{k}$ variables while $\gamma_{i k}^{\circ}$ is the observed variogram, i.e. the variogram of $Z_{i}$ and $Z_{k}$. The $\gamma_{i k}^{\mathrm{u}}$ can be calculated if at least 2 acquisitions of the same image $Z_{i}^{1}(x)$ and $Z_{i}^{2}(x)$ are available [14] using the formula:

$$
\gamma_{i j}(h)=\frac{1}{2 N_{\mathrm{p}}} \sum_{N_{\mathrm{p}}}\left[\left(Z_{i}^{1}(x)-Z_{i}^{1}(x+h)\right)\left(Z_{j}^{2}(x)-Z_{j}^{2}(x+h)\right)\right]
$$

In this study, the underlying variograms were quite similar to the observed variograms except for the nugget effect which almost disappears when we estimate $\gamma^{\mathbf{u}}$. This indicates that the nugget effect is a measure of the noise. This could be obtained directly from the $\gamma^{\circ}$ on a single acquisition. In figures 5 and 6 the variograms $\gamma^{\circ}$ of the average of two acquisitions are given. It is clear that the weights depend on the structure of the image via their variogram, so that they are generally not uniform, as used in common a priori smoothing. This point will be illustrated later. In addition, the kriging system uses, by construction, the available information in the vicinity of the estimated pixel $x_{0}$. This information is useful to predict the underlying $Y_{j}\left(x_{0}\right)$, as far as the correlation between $Y_{j}\left(x_{0}\right)$ and the available data is sufficient. This is the case when the variogram changes slowly on the range of distances involved in the neighbourhood.

The kriging variance for the estimation of $Y_{j}\left(x_{\mathrm{o}}\right)$ is calculated as:

$$
\sigma_{k}^{2}=\sum_{\alpha=1}^{n} \sum_{i=1}^{N}\left(\lambda_{i j}^{\alpha} \gamma_{i j}^{\mathrm{u}}\left(x_{\alpha}-x_{\mathrm{o}}\right)+\mu_{j j}\right)
$$


It is always positive and it is useful to select the size of the neighbourhood for the filter. The filter can be designed using only one variable to filter the noise of the same image. This method is called univariate kriging. Its main strength is to give an estimation $Y_{j}^{*}\left(x_{0}\right)$ using the observed data $Z_{j}\left(x_{\alpha}\right)$ having the same physical meaning as $Y_{j}$. When several variables are available, one can use all of them to filter each variable; this method is called cokriging. The kriging equations and the procedure is the same, except that cokriging involves more equations. Cokriging uses spatial knowledge as well as inter-variable relations. The weights of the filter will have high values for highly correlated variables and low values for poorly correlated variables. When performing a cokriging, one expects to increase the noise removal (as compared to univariate kriging). A counterpart is that a mixture of various signals is made, which is physically questionable.

4.3 SignAL TO NOISE RATIO. - It is interesting to measure the amount of available information in an image, and to compare it before and after filtering, in order to quantitatively appreciate the improvement of the image. This can be achieved by means of the signal to noise ratio $(S / N)$, defined as the ratio:

\section{variance of the signal \\ variance of the error}

The variance of the signal is simply the variance of the underlying image $\left(\sigma_{u}^{2}\right)$ obtained as the sill of the cross variogram between two acquisitions, or by the difference between the variance of a single acquisition and the nugget effect $C_{\mathrm{o}}$ when it can be fully assigned to the noise. For the raw (noisy) image, the variance of the error is the difference $C_{\mathbf{o}}-C_{\mathbf{u}}$, where $C_{\mathbf{u}}$ is the nugget effect of the underlying variogram (estimated from two separate acquisitions). In practice in the present case, we observe $C_{\mathbf{u}} \simeq 0$ so that we use $C_{\mathbf{o}}$, and a single acquisition can provide this information. For the filtered image, the variance of the error is just the kriging variance $\left(\sigma_{k}^{2}\right)$ given above in section 4.2.

We must point out that this definition of the $(S / N)$ ratio is more general than, but covers, the conventional $(S / N)$ used in signal processing, and calculated from the cross-correlation between two acquisitions, since it can be used in other conditions of image degradation than by noise.

4.4 MODEL FOR THE KRIGING AND THE COKRIGING. - To solve the kriging system, it is necessary to model the variograms $\gamma^{\mathrm{u}}$ and $\gamma^{\mathrm{o}}$. Indeed, the use of the estimated variograms would not guarantee the positivity of the kriging variance $\sigma_{k}^{2}$, and therefore would lead to absurd results.

In cokriging, the simple and cross variograms must be consistent with one another to ensure a positive kriging variance. Different models can be fitted for each of the variables and then this can be used to build the kriging system. But unfortunately this system does not give a positive definite system and testing this a priori is very difficult. So, a single model is fitted for all simple and cross variograms at the same time with different sills. The sills are taken as a matrix which is modified to fit all of the variograms at the same time while remaining positive definite $[15,16]$. The model is defined as a linear combination of variogram functions $g_{\mathrm{s}}$ as:

$$
\gamma_{i j}(h)=\sum_{s=0}^{N_{s}} \gamma_{i j}^{s}(h)=\sum_{s=0}^{N_{s}} b_{i j}^{s} g_{s}(h)
$$

where $N_{s+1}$ is the number of structures, $g_{s}$ is the variogram model specific with range and sill equal to 1 , and $b_{i j}^{s}$ is the coefficient of the $i, j^{\text {th }}$ variable pair corresponding to the $s^{\text {th }}$ structure.

At first the $b_{i j}^{s}$ are fitted with the limits $0 \leq b_{i j}^{s}<\infty$ and $\sum_{s} b_{i i}^{s}$ is the variance of the $i^{\text {th }}$ variable.

The cross variograms are to be fitted under the conditions:

$$
-\sqrt{b_{i i}^{s} b_{j j}^{s}} \leq b_{i j}^{s} \leq \sqrt{b_{i i}^{s} b_{j j}^{s}}
$$


Table III. - Correlation coefficient matrix of uranium images.

\begin{tabular}{|l|c|c|c|c|c|}
\hline & BF 59 & BF 74 & BF 89 & BF 112 & BF CHA \\
\hline BF 59 & 1.00 & & & & \\
BF 74 & 0.88 & 1.00 & & & \\
BF 89 & 0.78 & 0.76 & 1.00 & & \\
BF 112 & 0.44 & 0.46 & 0.45 & 1.00 & \\
BF CHA & 0.07 & 0.00 & -0.08 & 0.83 & 1.00 \\
\hline
\end{tabular}

The hull of the variogram is defined as:

$$
\text { hull }\left(\gamma_{i_{j}}(h)\right)= \pm \sum_{s=0}^{N_{s}} \sqrt{b_{i i}^{s} b_{j j}^{s} g_{s}(h)}
$$

and is represented on the right-hand side of figure $5 \mathrm{a}$ and 6.

The model is fitted on an interactive graphic system by giving only the number of structures, type and range for each structure. For our images, we use 2 different scales in addition to the nugget effect, modelled by cubic and spherical variograms, to account for the inflexion point apparent on the experimental variograms.

- Nugget effect: $\gamma(h)=C_{\mathrm{o}}$ for $h \neq 0$.

- Cubic model:

$$
\begin{gathered}
\gamma(h)=C(h / a)^{2}\left[7-(h / a)\left[8.75-(h / a)^{2}\left[3.50-0.75(h / a)^{2}\right]\right]\right] \text { for } h \in[0, a] \\
\gamma(h)=C \text { for } h \geq a
\end{gathered}
$$

- Spherical model:

$$
\begin{gathered}
\gamma(h)=C\left[1.5(h / a)-0.5(h / a)^{3}\right] \text { for } h \in[0, a] \\
\gamma(h)=C \text { for } h \geq a
\end{gathered}
$$

where $a$ and $C$ are the range and the variance (sill) of the variogram. The results of the models are compared to the variograms (Fig. 5 and 6 ).

It is important to point out here that a good fit between the experimental variogram and their model (mainly for the lowest values of $h$ ) is primordial for the quality of the linear filter, through the calculation of the weights $\lambda_{i j}^{\alpha}$ and of the parameters of the $S / N$ ratio $\left(\sigma_{k}^{2}, \sigma_{u}^{2} . C_{o}\right.$ and $\left.C_{u}\right)$.

\section{Application of the filtering to EELS images.}

In this section, we will present and comment the main results of the filtering procedure when applied to the uranium and terbium EELS images.

5.1 URANIUM IMAGES. - After studying the statistics and the correlation coefficients (we recall here that the correlation coefficient $\rho_{i j}$ between the variables $Z_{i}$ and $Z_{j}$ is defined as:

$$
\rho_{i j}=E\left[Z_{i}, Z_{j}\right] / \sqrt{\operatorname{var}\left(Z_{i}\right) \cdot \operatorname{var}\left(Z_{\jmath}\right)}
$$

we have $-1 \leq \rho_{i} \leq 1$ ) (Tab. III), the variables were classified into 3 groups for cokriging as below: 
- Group 1: BF59, BF74, BF89

- Group 2: BF112

- Group 3: BFCHA (characteristic image).

The images of the same group are strongly correlated comparatively. Image BF112 is on the uranium peak and hence its behaviour is different from the others of group 1. Even although the characteristic image is strongly correlated with BF112, it was separated because the images were not of the same type. Doing so, we expect in our procedure to improve the quality of the images, without mixing data with different physical contents.

All the structures were almost similar, with a cubic and spherical models. Only the first group has a long cubic structure and also the range of the first structure is higher than the others. In groups 2 and 3 , the first range is around 10 pixels. The clusters of atoms have approximately the same diameter.

Concerning the neighbourhood of each pixel to design the filter, various sizes were tried as shown in tables V and VI for the characteristic image of uranium. Because we use an isotropic model of variogram, only a part of the filter is given in table $\mathrm{V}$, and the remaining can be constructed by symmetry. The neighbours are numbered from 1 starting at the centre (Tab. IV). Depending on the position, each neighbour can occur either 1 or 4 or 8 times. From table V, it is clear that the weights of the optimal linear filters are non uniform: they decrease according to the distance to the central point $x_{\mathrm{o}}$. From the $5 \times 5$ size of the filter, there is almost no change of the distribution of the weights, the boundary weights being almost equal to zero. Similarly, the $S / N$ ratio of the filtered image increases with the filter size (Tab. VI). However, almost nothing is gained beyond the $5 \times 5$ size. As a consequence, we decided to use a $9 \times 9$ filter (almost the size of the range of the variogram model). Similar results would have been obtained with a $5 \times 5$ filter resulting in shortest calculations.

Table IV. - Location of the weights of the filters.

\begin{tabular}{|c|c|c|c|c|c|}
\hline 21 & 20 & 19 & 18 & 17 & 16 \\
\hline & 15 & 14 & 13 & 12 & 11 \\
\hline & & 10 & 9 & 8 & 7 \\
\hline & & & 6 & 5 & 4 \\
\hline & & & & 3 & 2 \\
\hline & & & & & 1 \\
\hline
\end{tabular}

For the group 1, the filter is designed using the cokriging method whereas for the other groups a single variable is used. The filtered images are shown together with the raw images to see how the filtering effect enhances the signal content. The image BF112 is on the uranium edge and hence one can clearly see some of the clusters in the filtered image. The group 1 images were used to estimate the background and hence do not contain any clusters.

The characteristic image and its results are shown in figure 7. Figure 7a shows the characteristic image constructed directly from the original BF images. The image (Fig. $7 \mathrm{~b}$ ) is the filtered image for figure 7a. At last the image (Fig. 7c) is constructed from the filtered BF images. The number of clusters and their boundaries are very clearly seen in figures $7 \mathrm{~b}$ and $7 \mathrm{c}$. 
Table V. - Filter weights for various size filters (uranium characteristic images).

\begin{tabular}{|c|c|c|c|c|}
\hline $3 \times 3$ & $5 \times 5$ & $7 \times 7$ & $9 \times 9$ & $11 \times 11$ \\
\hline 0.133 & 0.101 & 0.098 & 0.093 & 0.092 \\
\hline 0.113 & 0.075 & 0.073 & 0.069 & 0.068 \\
\hline \multirow[t]{19}{*}{0.103} & 0.059 & 0.058 & 0.056 & 0.055 \\
\hline & 0.035 & 0.038 & 0.037 & 0.037 \\
\hline & 0.026 & 0.030 & 0.031 & 0.030 \\
\hline & 0.004 & 0.013 & 0.017 & 0.017 \\
\hline & & 0.007 & 0.012 & 0.013 \\
\hline & & 0.003 & 0.009 & 0.010 \\
\hline & & -0.006 & 0.003 & 0.004 \\
\hline & & -0.017 & -0.003 & -0.001 \\
\hline & & & -0.003 & 0.000 \\
\hline & & & -0.004 & -0.001 \\
\hline & & & -0.006 & -0.002 \\
\hline & & & -0.008 & -0.004 \\
\hline & & & -0.008 & -0.004 \\
\hline & & & & -0.005 \\
\hline & & & & -0.005 \\
\hline & & & & -0.005 \\
\hline & & & & -0.004 \\
\hline & & & & -0.002 \\
\hline & & & & 0.002 \\
\hline 1.000 & 1.000 & 1.119 & 1.204 & 1.216 \\
\hline-0.000 & -0.000 & -0.119 & -0.204 & -0.216 \\
\hline 1.000 & 1.000 & 1.000 & 1.000 & 1.000 \\
\hline
\end{tabular}

The $S / N$ ratio is calculated for the raw images and also for the filtered images. The ratio is increased by 5 to 20 times (Tab. VII). We cannot calculate $S / N$ ratio of image of figure 7c, as $\sigma_{k}^{2}$ is unknown. However for this example, it seems that images in figure $7 \mathrm{~b}$ and $7 \mathrm{c}$ look equivalent and that the direct kriging of the noisy characteristic image gives satisfactory results.

5.2 Terbium IMAges. - The correlation matrix of original images is given in table VIIIa. The correlations are not very strong due to the presence of noise, but they are approximately of the same range and so, all are grouped together for the cokriging.

The experimental simple and cross variograms are calculated for the masked images (Fig. 6). The model is similar to the model of uranium. Unlike in uranium, here the long structure was not present in the model. One structure is cubic which is continuous at the origin with a range of 11 
Table VI. - S/N ratio for different size filters. $\sigma_{u}^{2}$ : variance of the signal. $\sigma_{k}^{2}$ : variance of estimation. $S / N=\sigma_{\mathrm{u}}^{2} / \sigma_{k}^{2}$.

Uranium characteristic image
\begin{tabular}{|c|c|c|}
\hline & $\sigma_{\mathrm{u}}^{2}=4739.55$ \\
\hline Original & 2849.97 & $S / N$ ratio \\
$3 \times 3$ & 583.62 & 1.66 \\
$5 \times 5$ & 443.79 & 10.68 \\
$7 \times 7$ & 429.02 & 11.05 \\
$9 \times 9$ & 408.38 & 11.61 \\
$10 \times 10$ & 401.81 & 11.79 \\
\hline
\end{tabular}

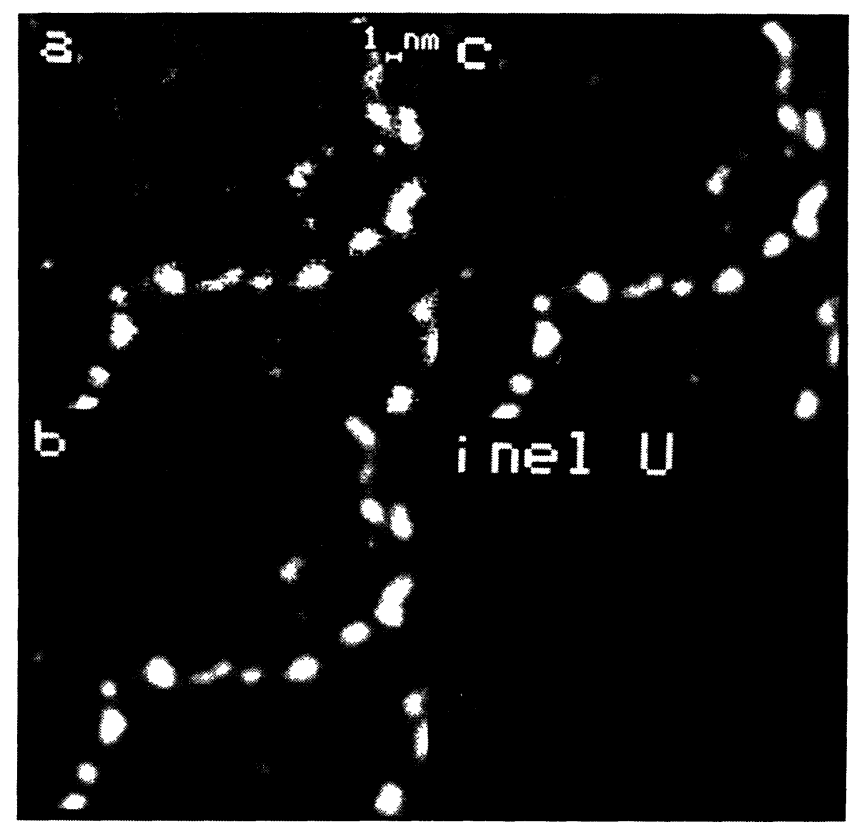

Fig. 7. - Uranium characteristic image: (a) constructed from original images; (b) filtered version of (a); (c) estimated from filtered images shown in figure 3 (right column).

and the other structure is spherical with a range of 20 .

The images are cokriged using a $9 \times 9$ neighbourhood. Because of the smaller correlations, the kriged variable has strong weights compared to the weights of the covariables. The correlation coefficients were calculated on the filtered images and shown in table VIIIb. Due to the large contribution of the central point weight $\lambda\left(x_{\mathrm{o}}\right)$, there is a very strong linear correlation between the filtered component images.

The signal to noise ratio values are shown in table IX. The original images are very noisy. The reduction of noise is very high (a 11-13 factor is observed). The original and filtered images of terbium BF images are shown in figure 4. The characteristic image constructed from these original images is shown in the bottom left part of figure 4. This image contains no information and its 
Table VII. - Comparison of the $S / N$ ratio for different size filters.

\begin{tabular}{|c|c|c|}
\hline Image & $\begin{array}{r}S / N \\
\text { Original }\end{array}$ & Filtered \\
\hline BF 59 & 13.97 & 71.27 \\
BF 74 & 5.43 & 56.26 \\
BF 59 & 13.97 & 71.27 \\
BF 89 & 2.39 & 41.59 \\
BF 112 & 2.73 & 15.30 \\
BF CHA & 1.08 & 11.61 \\
\hline
\end{tabular}

Table VIII. - Correlation coefficient matrix of original (a) and kriged (b) terbium images.

a)

\begin{tabular}{|c|c|c|c|} 
Original images \\
\begin{tabular}{|c|c|c|c|}
\hline Image & BF 131 & BF 141 & BF 151 \\
\hline BF 131 & 1.000 & 0.521 & 0.494 \\
\hline BF 141 & 0.521 & 1.000 & 0.489 \\
\hline BF 151 & 0.494 & 0.489 & 1.004 \\
\hline
\end{tabular}
\end{tabular}

\begin{tabular}{|c|c|c|c|} 
Kriged images \\
\hline Image & BF 131 & BF 141 & BF 151 \\
\hline BF 131 & 1.000 & 0.980 & 0.976 \\
\hline BF 141 & 0.980 & 1.000 & 0.978 \\
\hline BF 151 & 0.976 & 0.978 & 1.000 \\
\hline
\end{tabular}

variogram is only a pure nugget effect. On the other hand, the variogram of the bottom right part of figure 4, is similar to the variogram shown in figure $5 \mathrm{c}$, with a highest discontinuity at the origin, due to the residual noise in the filtered image.

Table IX. - Comparison of S/N ratio for the different original and filtered terbium images.

\begin{tabular}{|c|c|c|}
\hline Image & $S / N$ (raw) & $S / N$ (filt) \\
\hline BF 131 & 1.33 & 11.77 \\
\hline BF 141 & 1.00 & 13.52 \\
\hline BF 151 & 0.90 & 11.86 \\
\hline
\end{tabular}


As a consequence and contrarily to the uranium case, it is not possible to give a filtered version of this image. In this case we need the use of filtered BF images to restore the characteristic image. The image constructed from tiltered BF amages shows clearly clusters of atoms in the bottom right part of figure 4 . The effect of the tiiter is very efficient in this case. Also this suggests that the construction of characteristic images from filtered BF image is the best procedure in the case of very noisy BF images.

5.3 COKRIGING VERSUS UNIVARIATE KRIGING. - Each BF component of the uranium and terbium images can be filtered either separately (univariate kriging) or by use of all available information (cokriging). As the second approach of filtering is much more complex for implementation. it is instructive to compare the performances of the two procedures. This can be made by means of the $S / N$ ratio resulting from the kriging variances, and given in table $\mathrm{X}$. In each case, the $S / \mathrm{V}$ ratio is largely increased by the filter. This is even more important for the terbium, where the initial $S / N$ ratio is close to 1 , and for which the resulting characteristic image is practically a pure noise.

Table X. - Comparison of S/N ratio for the univariate and cokriging techniques.

\begin{tabular}{|c|c|c|c|c|}
\hline \multirow{2}{*}{ Chemical element } & \multirow{2}{*}{ Image } & \multicolumn{3}{|c|}{ Signal } \\
\cline { 3 - 5 } & & Original & Univariate & Cokriging \\
\cline { 2 - 5 } & BF 59 & 13.97 & 79.22 & 71.27 \\
Uranium & BF 74 & 5.43 & 42.57 & 56.26 \\
& BF 89 & 2.39 & 21.66 & 41.59 \\
& TB 131 & 1.33 & 10.11 & 11.77 \\
Terbium & TB 141 & 1.00 & 10.37 & 13.51 \\
& TB 151 & 0.90 & 9.54 & 11.86 \\
\hline
\end{tabular}

As expected, the cokriging procedure generally improves the $S / \mathrm{N}$ ratio, when compared to the univariate kriging, except in one case (uranium BF59), where the multivariate model of regionalisation is very different (and with a bad fit) as compared to the univariate model. For the uranium BF89 image, the gain is nearly a factor of 2 . As we are interested by the best final estimation of characteristic images, we did use cokriged images in this study. However very similar results, at the expense of a simpler procedure, would have been obtained from univariate kriging in the present case.

In addition, as already mentioned and seen from the correlation coefficients given in table VIII, the cokriged images are highly correlated. As a result, the coefficients $A$ and $R$ used to estimate the characteristic image remain certainly almost constant from pixel to pixel when working with the cokriged images, whereas they would depend on the local chemical composition when working with initial or univariate kriged images. If one wishes to avoid this situation. univariate kriging should be prefered, at the expense of a slight decrease of the final $\Xi, V$ ratio. but with advantage of displaying a "pure" chemical signal.

5.4 EM POINT RESOLUTION. - The EM point resolution [2] is equal to the distance at which the covariance is equal to the half of the maximum covariance. Consider $h^{\prime}$ is the EM resolution 
where:

$$
C\left(h^{\prime}\right)=\frac{C(0)}{2} \Rightarrow \gamma\left(h^{\prime}\right)=\frac{C(0)}{2}
$$

This supposes a linear transfer theory of image formation, in which case the width (finite width) of the peak contains correlations in the object and in the image forming system. When the noise is additive and uncorrelated with the signal, the observed variogram contains also the part of the noise component. So the half width of the variogram peak between pairs of duplicated images will be used as the EM point resolution. This definition is valid for incoherent optical systems and for coherent or partially coherent ones when the transmittance is weak.

In our case, the raw images have 2 acquisitions. So using these 2 acquisitions, the underlying variogram can be calculated and in turn used to define the EM resolution. From the present definition of resolution, no apparent change of resolution occurs while comparing the variograms from the initial and the filtered images. This is not the case when any other filter is utilized. This results from the fact that the kriging procedure looks for weights that minimize the variance of the error $\sigma_{k}^{2}$. This variance is a combination of the amount of noise in the image, and of the distorsion (involving a decrease of the resolution among others) produced by the filter. When $a$ priori smoothing filters are introduced (such as a common moving average), it is easy to see that the noise is removed, but simultaneously the resolution is lost when increasing the size of the filter, resulting in poor $S / N$ ratio [14]. The kriging, which is an adaptive filter, automatically finds the best compromise between the noise suppression and the loss of resolution for a given structure.

\section{Conclusion.}

The design of linear optimal unbiased filters to improve noisy EELS images was discussed and tested on uranium and terbium images. The main results of the application of a well established geostatistical technique to high resolution images are the following:

A very large improvement of the $S / N$ ratio (as calculated from the variance of estimation of the underlying images) can be achieved on each component of the EELS images, resulting into a significant enhancement of the small clusters seen on the characteristic images of uranium, and into the appearance of a structure on the characteristic image of terbium. The obtained $S / N$ ratio can be improved by a factor 5 to 10 .

In the present images $9 \times 9$ filter sizes were implemented. Similar results could be obtained by $5 \times 5$ filters, with a shorter calculation. It seems that the simplest version, based on a separate filtering of each BF image would give good results. In any case, no apparent degradation of the spatial resolution was observed on the filtered images.

As far as there is no structure with a range less than the distance between two adjacent pixels of the image, the procedure can be used with a single acquisition of the mappings, which makes the experiments simpler. Additional experiments on various types of EELS images are required to give definite conclusions on the ultimate resolution that can be achieved with the instrument after enhancement of the noisy images. Further developments could be included in the procedure, such as a deconvolution made by kriging [17] (to account for the shape of the electron probe) or the implementation of non linear optimal filters. 


\section{References}

[1] Castaing R., Henry L., C.R. Acad. Sci. Paris B255 (1962) 76.

[2] COLLIEX C. and MORY C., In Quantitative Electron Microscopy, J.N. Chapman and A.J. Craven (Eds.) Scottish Universities Summer School in Physics (1984) p. 149.

[3] MORY C., BonNet N., COllieX C., KoHl H. and TenCÉ M., Scanning Microscopy Supt. 2 (1988) p. 329.

[4] Jeanguillaume C., Trebbia P. and Colliex C., Ultramicroscopy 3 (1978) 237.

[5] Bonnet N., COllieX C., MORY C. and TeNCÉ M., Scanning Microscopy Supt 2 (1988) p. 351.

[6] MORY C. and COLLIEX C., Ultramicroscopy 28 (1989) 339.

[7] MORY C., KOHL H., TENCÉ M. and COllieX C., To be published in Ultramicroscopy (1991).

[8] WIENER N., Extrapolation, Interpolation and Smoothing of Stationary Time Series (M.I.T. Technol. Press and John, Wiley \& Sons, New York, 1949).

[9] PratT W.K., Digital Image Processings (John Wiley \& Sons, New York, 1978).

[10] TREBBiA P. and BONNET N., Ultramicroscopy 34 (1990) 165.

TREBBiA P. and MORY C., Ultramicroscopy 34 (1990) 179.

[11] MORY C., CollieX C., REVET B. and DELAIN E., Ultramicroscopy 7 (1981) 161.

[12] MATHERon G., Les variables régionalisées et leur estimation (Masson et $C^{\text {ie }}$ Ed., Paris, 1965).

[13] Journel A.G. and HuiJBregTs C.J., Mining geostatistics (Academic Press, 1978).

[14] DALY C., JEUlin D. and LAJAunie C., Application of multivariate kriging to the processing of noisy images, Proc. $3^{\text {rd }}$ Int. Geostat. Congress, Geostatistics, M. Armstrong Ed. (1989) pp. 749-760.

[15] BHANU PRASAD P., Multispectral linear filtering of high resolution EELS images by geostatistics. Report $\mathrm{n}^{0} 255$, Centre de Géostatistique, Fontainebleau (Paris School of Mines publication, 1989).

[16] WACKERNAGEL H., The inference of the linear model of the coregionalisation in the case of a geochemical data set. Sciences de la Terre, Série Informatique 24 (1985) 81.

[17] CONAN V., HOWARD C.W., JEULIN D., RENARD D., CUMMINS P., Improvements of 3D confocal microscope images by geostatistical filters, Trans. R. Microsc. Soc. (Adams Hilger, Bristol, Philadelphia, NY) 1 (1990) 281. 